3-1-1997

\title{
Bifurcation of The Equilibrium States of A Weightless Liquid Bridge
}

Lev A. Slobozhanin

J. Iwan D. Alexander

University of Alabama

Andrew Resnick

Cleveland State University, a.resnick@csuohio.edu

Follow this and additional works at: https://engagedscholarship.csuohio.edu/sciphysics_facpub

Part of the Physics Commons

How does access to this work benefit you? Let us know!

Publisher's Statement

(C) 1997 American Institute of Physics.

\section{Repository Citation}

Slobozhanin, Lev A.; Alexander, J. Iwan D.; and Resnick, Andrew, "Bifurcation of The Equilibrium States of A Weightless Liquid Bridge" (1997). Physics Faculty Publications. 241.

https://engagedscholarship.csuohio.edu/sciphysics_facpub/241

This Article is brought to you for free and open access by the Physics Department at EngagedScholarship@CSU. It has been accepted for inclusion in Physics Faculty Publications by an authorized administrator of

EngagedScholarship@CSU. For more information, please contact library.es@csuohio.edu. 


\title{
Bifurcation of the equilibrium states of a weightless liquid bridge ${ }^{a)}$
}

\author{
Lev A. Slobozhanin, ${ }^{\text {b) }}$ J. Iwan D. Alexander, and Andrew H. Resnick \\ Center for Microgravity and Materials Research, M-65 Research Institute Building, \\ University of Alabama in Huntsville, Huntsville, Alabama 35899
}

(Received 29 May 1996; accepted 28 March 1997)

\begin{abstract}
The bifurcation of the solutions of the nonlinear equilibrium problem of a weightless liquid bridge with a free surface pinned to the edges of two coaxial equidimensional circular disks is examined. The bifurcation is studied in the neighborhood of the stability boundary for axisymmetric equilibrium states with emphasis on the boundary segment corresponding to nonaxisymmetric critical perturbations. The first approximations for the shapes of the bifurcated equilibrium surfaces are obtained. The stability of the bifurcated states is then determined from the bifurcation structure. Along the maximum volume stability limit, depending on values of the system parameters, loss of stability with respect to nonaxisymmetric perturbations results in either a jump or a continuous transition to stable nonaxisymmetric shapes. The value of the slenderness at which a change in the type of transition occurs is found to be $\Lambda_{s}=0.4946$. Experimental investigation based on a neutral buoyancy technique agrees with this prediction. It shows that, for $\Lambda<\Lambda_{s}$, the jump is finite and that a critical bridge undergoes a finite deformation to a stable nonaxisymmetric state. $\neg$ (C) 1997 American Institute of Physics. [S1070-6631(97)02507-5]
\end{abstract}

\section{INTRODUCTION}

Liquid bridge statics problems have been studied since the early work of Plateau ${ }^{1}$ and are still of interest. ${ }^{2,3}$ Liquid bridges are important factors when considering liquid management under low-gravity conditions. Perhaps the most studied configurations involve bridges held between disks or rods. These are used to analyze problems associated with zone melting under zero-gravity (see, for example, Refs. 2 and 4).

This paper concerns the bifurcation of equilibrium shapes of a weightless liquid bridge with its free surface pinned to the edges of coaxial equidimensional circular supports (radius $a$ ) separated by a distance $2 h$. The supports can be considered as solid disks without loss of generality (see Fig. 1). In this case, the equilibrium state of the liquid is determined by two parameters: the slenderness $\Lambda$ and the relative volume $V$ :

$$
\left.\Lambda=h / a,\urcorner \quad V=v /\left(2 \pi a^{2} h\right),\right\urcorner
$$

where $v$ is the actual liquid volume supported between the disks.

The shape and stability of axisymmetric liquid bridge configurations has been studied extensively. For zero-gravity conditions, the equilibrium surface is a surface of constant mean curvature and, for the axisymmetric case, the bridge can have a cylindrical, spherical, catenoidal, unduloidal, or nodoidal shapes. Typically, previous investigations of weightless bridge stability have assumed that the perturbations satisfy the constraints of constant liquid volume and fixed contact lines. We summarize such work below.

It has been established theoretically ${ }^{1}$ and experimentally $^{1,5}$ that a cylindrical bridge is stable if $\Lambda<\pi$ and

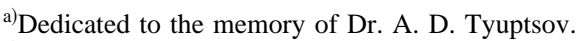

${ }^{b}$ On leave from the B. Verkin Institute for Low Temperature Physics and Engineering, National Academy of Sciences of Ukraine. Electronic address: lion@cmmr.uah.edu
}

unstable if $\Lambda>\pi$. Here the critical perturbation is axisymmetric. ${ }^{1,6}$ It was suggested by Gillette and Dyson ${ }^{7}$ that, when $V=1$, there are no stable noncylindrical axisymmetric surfaces. Rivas and Meseguer ${ }^{8}$ determined the linear dependence of $\Lambda$ on $V$ for critical unduloids that are close to a cylinder.

Constant volume spherical bridges are always stable. This follows from Plateau's experimental results and is easily proved theoretically (see, for example, Refs. 9 and 10).

Plateau determined the region of existence for catenoidal bridges experimentally. A theoretical and experimental analysis of the stability of catenoidal bridges with respect to axisymmetric perturbations was later carried out by Erle et al. ${ }^{11}$

Besides cylinders, spheres, and catenoids, Plateau ${ }^{1}$ also undertook experimental investigations of the stability of unduloidal and nodoidal bridge surfaces and qualitatively described the results. For axisymmetric perturbations and arbitrary values of $\Lambda$ and $V$, the stability limits were first constructed by Gillette and Dyson ${ }^{7}$ on the basis of Howe's theory $^{12}$ (outlined in Refs. 3, 7, and 11). They also proved that an axisymmetric bridge with no equatorial symmetry plane is always unstable. Furthermore, they later proved ${ }^{13}$ that axisymmetric perturbations are the most dangerous for weightless bridges that are axisymmetric about the $z$ axis and have surfaces represented by single-valued functions $r$ $=r(z)$. Slobozhanin ${ }^{9}$ analyzed the stability of an axisymmetric bridge with respect to arbitrary (i.e., both nonaxisymmetric and axisymmetric) perturbations and constructed the general stability boundary in the $(\Lambda, V)$-plane. (These results are presented in English in Ref. 10, and the stability boundary is reproduced in Refs. 3 and 14.) Quantitative experimental data on the boundary of the stability region were obtained by Elagin et al. ${ }^{15}$ ("microzone" method), and by Sanz and Martínez ${ }^{16}$ and Russo and Steen ${ }^{17}$ (neutral buoyancy technique).

The conditions for which capillary surfaces with contact 


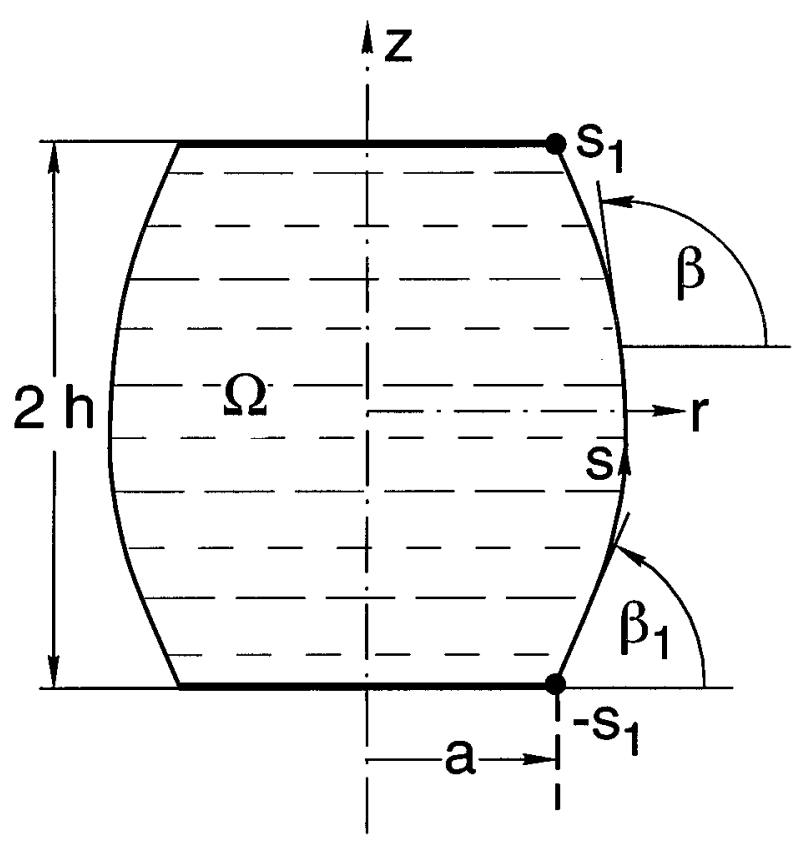

FIG. 1. Geometry of the equilibrium system.

lines pinned to solid edges are most unstable to perturbations of the liquid surface, rather than to perturbations of the contact line, were obtained in a more general analysis by Slobozhanin and Tyuptsov ${ }^{18}$ (see also Ref. 10). This was also examined for the particular cases of liquid bridges held between disks and rods by Slobozhanin. 9,19

If an axisymmetric equilibrium state is stable, then, for a small variation of the parameters $\Lambda$ and $V$, it has a unique continuous extension and the stability of the state is preserved. However, if the equilibrium state lies on the stability boundary, the uniqueness of the continuous extension is violated and the equilibrium state bifurcates. Methods for analyzing bifurcations of the equilibrium states of a capillary liquid mass and the possible bifurcation structures have been described in earlier work (see, for example, Refs. 3, 10, and 20-23).

To account for Plateau's experimental results, Michael $^{24}$ proposed possible bifurcation patterns that are plausible when the different axisymmetric equilibrium shapes of a bridge lose their stability. He further emphasized the need to study the corresponding bifurcation problem in detail. This problem has been solved for a critical cylinder. The solution is a particular case of results obtained by Brown and Scriven $^{20}$ and by Vega and Perales. ${ }^{25}$ The dynamical behavior of an axisymmetric liquid bridge as it loses stability on the boundary segment along which axisymmetric perturbations are critical was studied in Refs. 26 and 27. Finally, a sophisticated analysis of the nature of the axisymmetric bifurcations along this boundary segment was made in a recent paper by Lowry and Steen. ${ }^{3}$

In this paper we focus attention on bifurcations along a stability boundary segment where nonaxisymmetric perturbations are critical. In Sec. II we outline previous stability results for initially axisymmetric bridges. Then we consider small perturbations of equilibrium surfaces (Sec. III) and describe a procedure to obtain a first approximation to the shapes of the bifurcated surfaces (Sec. IV). The bifurcation equation is given in Sec. V. In Sec. VI the results of numerical and analytical analyses are presented. Experimental results for a particular case analyzed in Sec. VI A are presented in Sec. VII.

\section{CRITICAL AXISYMMETRIC STATES}

In this section we present a summary of results previously obtained in Refs. 9 and 10. These results are needed to clearly define the stability region under consideration and are used as a starting point for the bifurcation analysis described in the subsequent sections.

\section{A. Axisymmetric shapes}

The equilibrium surface of an axisymmetric liquid bridge has a parametric representation $r(s), z(s)$, where $r$, $\varphi$, and $z$ are the cylindrical coordinates and $s$ is the arc length of any axial section $\varphi=$ const. The dependent variables $r$ and $z$ are then given by the solutions of the following ordinary differential equations:

$$
r^{\prime \prime}=-z^{\prime}\left(q_{0}-z^{\prime} / r\right), \neg \quad z^{\prime \prime}=r^{\prime}\left(q_{0}-z^{\prime} / r\right) \quad\left({ }^{\prime}=\frac{d}{d s}\right) .
$$

Here $q_{0}$ is twice the mean curvature of the surface. Thus, $q_{0}-z^{\prime} / r=\beta^{\prime}$, where $\beta=\beta(s)$ is the angle between the positive $r$-axis direction and the tangent to the axial profile directed in the sense of increasing $s$. The system (2) is invariant under the transformation $q_{0} \rightarrow-q_{0}, s \rightarrow-s$. For definiteness, it is assumed hereafter that $q_{0} \geqslant 0$. If $q_{0} \neq 0$, then the relations

$$
\rho=q_{0} r, \quad \zeta=q_{0} z, \quad \tau=q_{0} s \neg
$$

are used to transform the system (2) to the following form:

$$
\rho^{\prime \prime}=-\zeta^{\prime}\left(1-\zeta^{\prime} / \rho\right), \quad \zeta^{\prime \prime}=\rho^{\prime}\left(1-\zeta^{\prime} / \rho\right) \quad\left({ }^{\prime}=\frac{d}{d \tau}\right) .
$$

Critical, as well as stable, axisymmetric surfaces always have an equatorial symmetry plane, $z=0$. All possible axisymmetric shapes (except catenoids) with an equatorial symmetry plane are described by solutions of (4) with

$$
\rho(0)=\rho_{0}, \quad \rho^{\prime}(0)=0, \quad \zeta(0)=0, \quad \zeta^{\prime}(0)=1,
$$

or

$$
\rho(0)=\rho_{0}, \quad \rho^{\prime}(0)=0, \quad \zeta(0)=0, \quad \zeta^{\prime}(0)=-1
$$

as initial conditions at the equatorial point $\tau=0$. Solutions of (4) corresponding to boundary conditions (5) or (6) have, respectively, the forms

$$
\begin{aligned}
& \rho=\left(1+\mu_{1}^{2}+2 \mu_{1} \cos \tau\right)^{1 / 2}, \\
& \zeta=\int_{0}^{\tau} \frac{1}{\rho(\xi)}\left(1+\mu_{1} \cos \xi\right) d \xi,
\end{aligned}
$$

and 


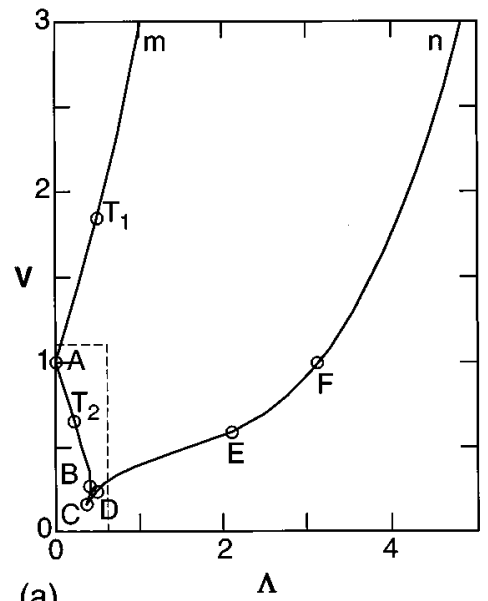

(a)

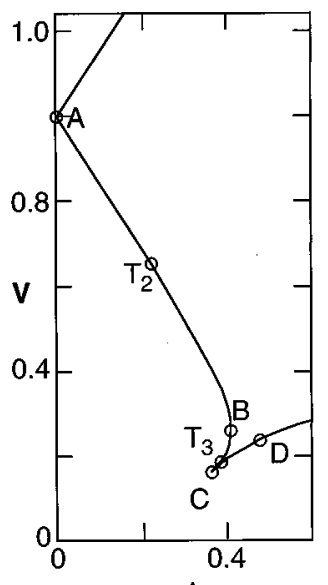

(b)

$\Lambda$

FIG. 2. Boundary of the stability region of an axisymmetric bridge under spatial perturbations for which the contact lines remain on the edges of the solids: (a) the general diagram and (b) the segment $A B C D$ of the stability boundary.

$$
\begin{aligned}
& \rho=\left(1+\mu_{2}^{2}-2 \mu_{2} \cos \tau\right)^{1 / 2}, \\
& \zeta=\int_{0}^{\tau} \frac{1}{\rho(\xi)}\left(1-\mu_{2} \cos \xi\right) d \xi,
\end{aligned}
$$

where $\mu_{1}=\rho_{0}-1$ and $\mu_{2}=\rho_{0}+1$.

\section{B. Stability for fixed contact lines}

We consider critical axisymmetric shapes for volumepreserving perturbations that leave the contact lines pinned to the disk edges. The corresponding boundary of the stability region is reproduced in Fig. 2. There are several special points on the stability boundary. Characteristics of liquid bridges corresponding to these points are listed in Table I. Except for $\Lambda$ and $V$, this table contains data for related initial conditions, the ratio $\rho_{0} / \rho\left(\tau_{1}\right)$ between equatorial and disk radii, and the angle of inclination $\beta_{1}$ at the lower disk (Fig. 1). Table II summarizes qualitative properties of the critical surfaces and the associated critical perturbations. Both tables generalize related results presented in Ref. 7. In addition, typical shapes of critical bridge profiles are shown in Fig. 3.

Profiles of critical bridges from the stability boundary segments $m A B C$ and $E F n$ have, respectively, horizontal and vertical tangents at their endpoints. The component of dan- gerous perturbations normal to axisymmetric equilibrium surfaces is proportional to $\zeta^{\prime}(\tau) \sin \varphi$ (on $m A B C$ ), to $\rho^{\prime}(\tau)$ (on $E F n$ ) and to $\sin \tau$ (at the point $F$ ). Along the segment $C D E$, the dangerous perturbations are axisymmetric ones with an equatorial symmetry plane. With the exception of the point $D$, the terminal point $\tau_{1}$ of a critical profile on $C D E$ is defined as that value of $\tau$ for which the function

$$
\bar{\phi}(\tau)=\bar{u}_{0}(\tau) \int_{0}^{\tau} \rho \bar{u}_{1}(\tau) d \tau-\bar{u}_{1}(\tau) \int_{0}^{\tau} \rho \bar{u}_{0}(\tau) d \tau
$$

first changes its sign. Here $\overline{u_{0}}(\tau)$ and $\overline{u_{1}}(\tau)$ satisfy

$$
\begin{aligned}
& \left.\left.\left.\bar{L}_{0} \overline{u_{0}}=0,\right\urcorner \bar{u}_{0}(0)=1,\right\urcorner \vec{u}_{0}(0)=0,\right\urcorner \\
& \left.\left.\left.\bar{L}_{0} \bar{u}_{1}=1,\right\urcorner \bar{u}_{1}(0)=1,\right\urcorner \vec{u}_{1}(0)=0,\right\urcorner
\end{aligned}
$$

with

$$
\overline{L_{0}} \bar{u} \equiv \vec{u}^{\prime \prime}+\frac{\rho^{\prime}}{\rho} \vec{u}+\left[\frac{\zeta^{\prime 2}}{\rho^{2}}+\left(1-\frac{\zeta^{\prime}}{\rho}\right)^{2}\right] \bar{u} .
$$

There are several useful approximate expressions for the relative volume $V(\Lambda)$ of the critical states. For small $\Lambda$, we consider (7) and (8), use series expansions in powers of the small parameter $\nu=\rho_{0}^{-1}$, and then eliminate $\nu$ to obtain

$$
\begin{aligned}
& \left.V=1+\Lambda \frac{\pi}{2}+\Lambda^{2}\left(\frac{8}{3}-\frac{1}{4} \pi^{2}\right)+o\left(\Lambda^{2}\right),\right\urcorner \\
& V=1-\Lambda \frac{\pi}{2}+\Lambda^{2}\left(\frac{8}{3}-\frac{1}{4} \pi^{2}\right)+o\left(\Lambda^{2}\right)
\end{aligned}
$$

at the points on $A m$ and $A B$, respectively. For critical states close to the critical cylindrical one, we expand (7) in powers of $\mu_{1}=\rho_{0}-1$. This yields the approximation

$$
\left.V=1+2\left(\frac{\Lambda}{\pi}-1\right)+\frac{5}{2}\left(\frac{\Lambda}{\pi}-1\right)^{2}+o\left(\left|\frac{\Lambda}{\pi}-1\right|^{2}\right),\right\urcorner
$$

which is more precise than the linear expression obtained

\begin{tabular}{|c|c|c|c|c|c|c|}
\hline Point $\neg$ & $\mathrm{IC}^{\mathrm{a}}$ & $\rho_{0}$ & $\rho_{0} / \rho\left(\tau_{1}\right)$ & $\beta_{1}(\mathrm{deg}) \neg$ & $\Lambda$ & V \\
\hline$T_{1}$ & (5) & ר3.787 & ר1.456 & ר0 & ר0.495 & 1.851 \\
\hline$A \neg$ & $\ldots$ & $\infty$ & $1 \neg$ & $\ldots$ & 0 & 1 \\
\hline$T_{2}$ & (6) & ר2.659 & ר0.755 & ר180 & ר.223 & 0.655 \\
\hline$B\urcorner$ & (6) & ר0.305 & ר0.364 & ר180 & ר0.405 & 0.263 \\
\hline$T_{3}$ & (6) & ר0.139 & ר0.255 & ר180 & ר.383 & 0.189 \\
\hline$C\urcorner$ & (6) & ר0.095 & רס213 & ר180 & $0.361 \neg$ & 0.164 \\
\hline$D\urcorner$ & $\cdots$ & ר0 & ר0.2107 & ר167.84 & רס 0.4718 & 0.2405 \\
\hline$E \neg$ & (5) & ר0.589 & ר0.417 & ר90 & ר2.128 & 0.591 \\
\hline$F \neg$ & (5) & 1 & 1 & $\neg 90$ & $\pi$ & 1 \\
\hline
\end{tabular}
earlier by Rivas and Meseguer. ${ }^{8}$ A comparison with numerical results showed that the relative error of our approximations is less than $1 \%$, provided that $\Lambda$ satisfies $\Lambda \leqslant 0.44, \Lambda$ $\leqslant 0.23$, and $2.5 \leqslant \Lambda \leqslant 4$ in Eqs. (12), (13), and (14), respectively. Finally, in the limit $\Lambda \rightarrow \infty$, the asymptotic representation $V \sim(2 / 3) \Lambda^{2}$ is valid along the nonintersecting curves $A m$ and $F n$.

TABLE I. Parameters of critical bridges at distinctive points of the stability boundary.

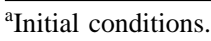


TABLE II. Characteristics of boundary segments.

\begin{tabular}{|c|c|c|c|c|c|c|}
\hline $\begin{array}{c}\text { Open } \\
\text { segment }\end{array}$ & $\begin{array}{l}\text { Critical } \\
\text { surface } \neg\end{array}$ & IC & $\rho_{0}$ & $\left.\beta_{1}(\operatorname{deg})\right\urcorner$ & $\mathrm{CP}^{\mathrm{a}}$ & $\begin{array}{c}\text { Type of } \\
\text { bifurcations }\end{array}$ \\
\hline$m T_{1}$ & $\begin{array}{l}\text { Bulged } \neg \\
\text { nodoid } \neg\end{array}$ & (5) & $(2,3.787)$ & ᄀ0 & non-ax. ${ }^{b}$ & $\begin{array}{l}\text { Supercritical } \\
\text { pitchfork }\end{array}$ \\
\hline$\left.T_{1} A\right\urcorner$ & $\begin{array}{l}\text { Bulged } \neg \\
\text { nodoid } \neg\end{array}$ & (5) & $(3.787, \infty)$ & ᄀ0 & nox-ax. ${ }^{b}$ & $\begin{array}{l}\text { Subcritical } \\
\text { pitchfork }\end{array}$ \\
\hline$A T_{2}$ & $\begin{array}{l}\text { Constricted } \neg \\
\text { nodoid } \neg\end{array}$ & (6) & $(2.659, \infty)$ & ר180 & non-ax. ${ }^{b}$ & $\begin{array}{l}\text { Subcritical } \\
\text { pitchfork }\end{array}$ \\
\hline$T_{2} B T_{3}$ & $\begin{array}{l}\text { Constricted } \neg \\
\text { nodoid } \neg\end{array}$ & (6) & $(0.139,2.659)$ & ר180 & non-ax. ${ }^{b}$ & $\begin{array}{l}\text { Supercritical } \\
\text { pitchfork }\end{array}$ \\
\hline$\left.T_{3} C\right\urcorner$ & $\begin{array}{l}\text { Constricted } \neg \\
\text { nodoid } \neg\end{array}$ & (6) & $(0.095,0.139)$ & ר180 & non-ax. ${ }^{b}$ & $\begin{array}{l}\text { Subcritical } \\
\text { pitchfork }\end{array}$ \\
\hline$C D \neg$ & $\begin{array}{l}\text { Constricted } \neg \\
\text { nodoid } \neg\end{array}$ & (6) & $(0,0.095)$ & $(167.8,180)$ & ax.-r.s. ${ }^{c}$ & $\begin{array}{l}\text { Subcritical } \\
\text { turning point }\end{array}$ \\
\hline Point $D \neg$ & Catenoid $\neg$ & $\cdots$ & ᄀ0 & ר167.8 & ax.-r.s. ${ }^{\mathrm{c}}$ & $\begin{array}{l}\text { Subcritical } \\
\text { turning point }\end{array}$ \\
\hline$D E \neg$ & $\begin{array}{l}\text { Constricted } \neg \\
\text { unduloid } \neg\end{array}$ & (5) & $(0,0.589)$ & $(90,167.8)$ & ax.-r.s. ${ }^{\mathrm{c}}$ & $\begin{array}{l}\text { Subcritical } \\
\text { turning point }\end{array}$ \\
\hline$E F \neg$ & $\begin{array}{l}\text { Constricted } \neg \\
\text { unduloid } \neg\end{array}$ & (5) & $(0.589,1)$ & ᄀ90 & ax.-r.a. ${ }^{\mathrm{d}}$ & $\begin{array}{l}\text { Subcritical } \\
\text { pitchfork }\end{array}$ \\
\hline Point $F \neg$ & Cylinder $\neg$ & (5) & $1 \neg$ & ר90 & ax.-r.a. ${ }^{\mathrm{d}}$ & $\begin{array}{l}\text { Subcritical } \\
\text { pitchfork }\end{array}$ \\
\hline$F n \neg$ & $\begin{array}{l}\text { Bulged } \neg \\
\text { unduloid } \neg\end{array}$ & (5) & $(1,2)$ & ר90 & ax.-r.a. ${ }^{\mathrm{d}}$ & $\begin{array}{l}\text { Subcritical } \\
\text { pitchfork }\end{array}$ \\
\hline
\end{tabular}

${ }^{\mathrm{a} C \text { Critical perturbations. }}$

${ }^{\text {b}}$ Nonaxisymmetric.

${ }^{c}$ Axisymmetric, reflectively symmetric about the equatorial plane.

${ }^{\mathrm{d}}$ Axisymmetric, reflectively antisymmetric about the equatorial plane.

\section{SMALL PERTURBATIONS}

In the general case, an equilibrium free surface under weightlessness is a surface of constant mean curvature $H$, i.e.,

$$
2 H=q .\urcorner
$$

Consider an axisymmetric equilibrium surface $\Gamma$ that encloses the domain $\Omega$ occupied by the liquid and a perturbed equilibrium surface close to $\Gamma$. Points $\mathbf{R}(s, \varphi)$ on the unperturbed surface are related to points $\mathbf{R}_{1}(s, \varphi)$ on the perturbed surface through a displacement, $N$, along the normal $\mathbf{n}$ to $\Gamma$, that is,

$$
\begin{aligned}
& \mathbf{R}_{1}(s, \varphi)=\mathbf{R}(s, \varphi)+\mathbf{n}(s, \varphi) N(s, \varphi), \\
& \mathbf{R}(s, \varphi)=\mathbf{i} r(s) \cos \varphi+\mathbf{j} r(s) \sin \varphi+\mathbf{k} z(s), \\
& \mathbf{n}(s, \varphi)=-\mathbf{i} z^{\prime}(s) \cos \varphi-\mathbf{j} z^{\prime}(s) \sin \varphi+\mathbf{k} r^{\prime}(s) .
\end{aligned}
$$

According to (16), for a point on the perturbed surface lying outside $\Omega, N$ will be positive (negative) if the unperturbed liquid domain $\Omega$ is to the right (left) as $s$ increases. The mean curvature of the perturbed surface can be calculated from the first and second fundamental forms of the surface ${ }^{28}$ using (16). It is a rather complicated expression and is presented, up to terms of third order in $N$, in Appendix A.

For a fixed slenderness, $\Lambda$, we let a perturbation of the equilibrium surface be induced by a small variation of the relative volume $\Delta V=V-V_{0}$. Here $V_{0}$ corresponds to the unperturbed volume. Then we require that $N(s, \varphi)$ should satisfy (15), the fixed contact line conditions

$$
N\left(-s_{1}, \varphi\right)=N\left(s_{1}, \varphi\right)=0,
$$
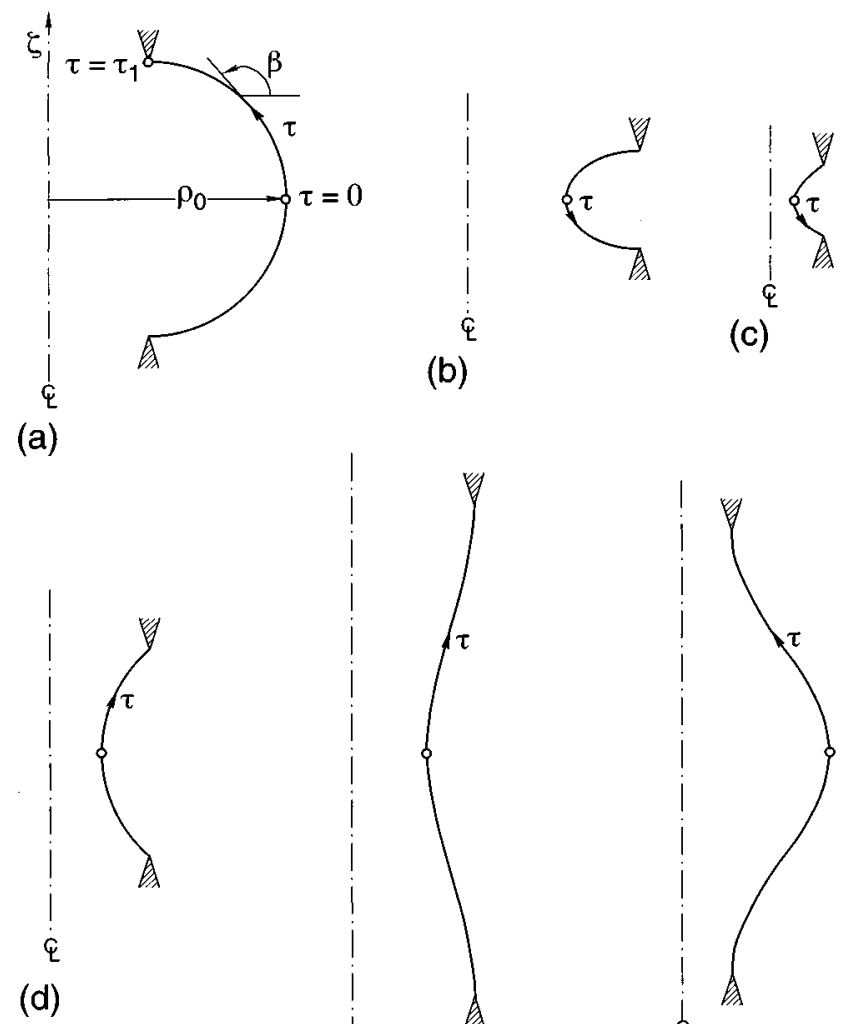

\section{(b)}

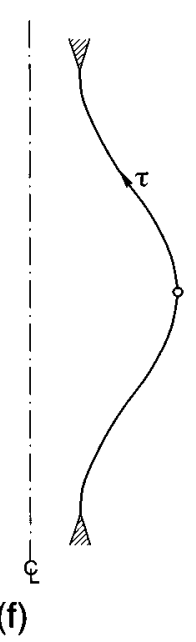

(e)
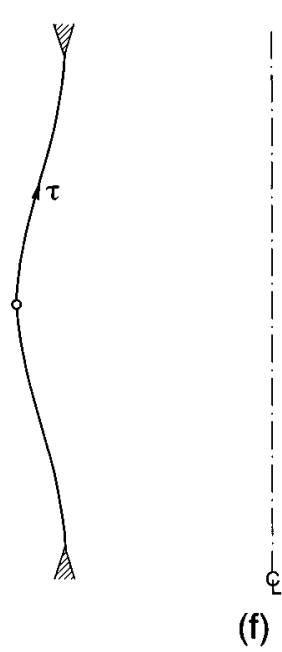

FIG. 3. Schematic sketch of the profiles of the critical axisymmetric equilibrium surfaces belonging to segments (a) $A m$, (b) $A B C$, (c) $C D$, (d) $D E$, (e) $E F$, and (f) $F n$ of the boundary of the stability region. 
and

$$
\begin{aligned}
\Delta V= & \pm \frac{1}{2 \pi a^{2} h} \int_{\Gamma}\left[N-\frac{1}{2}\left(\beta^{\prime}+\frac{z^{\prime}}{r}\right) N^{2}\right. \\
& \left.\left.+\frac{1}{3} \beta^{\prime} \frac{z^{\prime}}{r} N^{3}\right] d \Gamma .\right\urcorner
\end{aligned}
$$

Here $s=s_{1}$ and $s=-s_{1}$ are the values of $s$ at the endpoints of the profile of $\Gamma$. In (18), one should use the upper (lower) sign if $\Omega$ is to the right (left) as the arclength $s$ increases.

The solutions of the equilibrium problem bifurcate for critical values of the parameters. Therefore, in what follows the critical axisymmetric surface (with $V_{0}=V_{*}$ ) will be chosen as the surface $\Gamma$. To simplify the calculations, we shall assume $a$ priori that the rough case of bifurcation, i.e., where the bifurcated solutions are expanded in half-integral powers of a small increment of $V$, is the most typical for the critical states. ${ }^{10}$ The validity of this assumption will be established in Sec. VI C. Thus, we take

$$
\begin{aligned}
& V=V_{*} \pm \varepsilon^{2}, \neg \varepsilon>0 \\
& N=\varepsilon N_{1}(s, \varphi)+\varepsilon^{2} N_{2}(s, \varphi)+\cdots, \\
& q=q_{0}+\varepsilon q_{1}+\varepsilon^{2} q_{2}+\cdots .
\end{aligned}
$$

Here the choice of the upper or lower sign corresponds to an increase or decrease of the relative volume from its critical value.

Substituting (19) into (15), (17), and (18), and equating coefficients of like powers of $\varepsilon$, we find the following zerothorder relation for the critical axisymmetric surface $\Gamma$,

$$
\left.\beta^{\prime}+z^{\prime} / r=q_{0},\right\urcorner
$$

together with a sequence of boundary-value problems from which $N_{i}(s, \varphi)$ and $q_{i}(i \geqslant 1)$ can be determined. Using (20) and expression (A1) for $2 H$ (Appendix A), we can write the problems for $i=1,2$ and the differential equation for $i=3$ in the form

$$
\begin{aligned}
& \left.L N_{1}-q_{1}=0,\right\urcorner N_{1}\left(-s_{1}, \varphi\right)=N_{1}\left(s_{1}, \varphi\right)=0, \\
& \left.\int_{\Gamma} N_{1} d \Gamma=0,\right\urcorner \\
& \left.L N_{2}-q_{2}=f_{2}\left(r, z, N_{1}\right),\right\urcorner \\
& N_{2}\left(-s_{1}, \varphi\right)=N_{2}\left(s_{1}, \varphi\right)=0, \\
& \left.\int_{\Gamma} N_{2} d \Gamma=\frac{1}{2} q_{0} \int_{\Gamma} N_{1}^{2} d \Gamma \pm 2 \pi r^{2}\left(s_{1}\right)\left|z\left(s_{1}\right)\right|,\right\urcorner \\
& \left.L N_{3}-q_{3}=f_{3}\left(r, z, N_{1}, N_{2}\right),\right\urcorner
\end{aligned}
$$

where

$$
L N \equiv N_{s s}^{\prime \prime}+\frac{r^{\prime}}{r} N_{s}^{\prime}+\frac{1}{r^{2}} N_{\varphi \varphi}^{\prime \prime}+\left(\beta^{\prime 2}+\frac{z^{\prime 2}}{r^{2}}\right) N
$$

and the expressions for $f_{2}$ and $f_{3}$ are presented in Appendix A. We note that a prime superscript on the function $N$ denotes a partial derivative with respect to the argument indicated in the subscript.
It should be noted that, when the second term on the right-hand side of (24) is preceded by the upper sign, it represents an increase (decrease) of the relative volume, if with increasing $s$, the domain $\Omega$ is on the right (left). Alternatively, the lower sign represents a volume decrease (increase). One can see from Fig. 3 that the domain $\Omega$ is on the right when the critical surfaces belong to the $A B C D$ segment, and is on the left when critical surfaces belong to the $A m$ and $D E F n$ segments of the boundary.

\section{A FIRST APPROXIMATION}

For critical equilibrium states, the problem (21) has a nontrivial solution. The solution $N_{1}(s, \varphi)$ represents the shape of the perturbation that results in neutral stability. Taking into account the results that were presented earlier in Sec. II, we have

$$
\begin{aligned}
& \left.N_{1}=Q_{1} z^{\prime}(s) \sin \varphi, \quad q_{1}=0 \quad \text { on } \quad m A B C ;\right\urcorner \\
& \left.N_{1}=Q_{1} u(s), \neg q_{1}=Q_{1} \quad \text { on }\right\urcorner C D E ; \neg \\
& N_{1}=Q_{1} r^{\prime}(s), \\
& \left.q_{1}=0 \quad \text { on } E F n \text { except at the point } F ;\right\urcorner \\
& \left.N_{1}=Q_{1} \sin \frac{\pi z}{h},\right\urcorner q_{1}=0 \quad \text { at } F . \quad \neg
\end{aligned}
$$

Here we see that $N_{1}$ has nonaxisymmetric shapes along $m A B C$, while for the remaining portions of the stability boundary it is axisymmetric. The function $u(s)$ is even and, in the interval $0 \leqslant s \leqslant s_{1}$, is the solution of the problem

$$
\left.\left.\left.L_{0} u=1\right\urcorner\left(0 \leqslant s \leqslant s_{1}\right),\right\urcorner u\left(s_{1}\right)=0,\right\urcorner \int_{0}^{s_{1}} r u d s=0,
$$

and can be represented as

$$
\left.u(s)=u_{1}(s)+\alpha_{1} u_{0}(s) .\right\urcorner
$$

Here $\alpha_{1}=-u_{1}\left(s_{1}\right) / u_{0}\left(s_{1}\right)$, and $u_{0}(s)$ and $u_{1}(s)$ are the solutions of

$$
\begin{aligned}
& \left.\left.\left.\left.L_{0} u_{0}=0\right\urcorner\left(0 \leqslant s \leqslant s_{1}\right),\right\urcorner u_{0}(0)=1,\right\urcorner u_{0}^{\prime}(0)=0,\right\urcorner \\
& \left.\left.\left.\left.L_{0} u_{1}=1\right\urcorner\left(0 \leqslant s \leqslant s_{1}\right),\right\urcorner u_{1}(0)=1,\right\urcorner u_{1}^{\prime}(0)=0 .\right\urcorner
\end{aligned}
$$

The operator $L_{n}$ is given by

$$
L_{n} \equiv \frac{d^{2}}{d s^{2}}+\frac{r^{\prime}}{r} \frac{d}{d s}+\left(\beta^{\prime 2}+\frac{z^{\prime 2}}{r^{2}}-\frac{n^{2}}{r^{2}}\right), \neg n \geqslant 0 .
$$

For solutions of the form (31), the first of the conditions (30) is satisfied automatically, while the second is satisfied by the location of the endpoint $s_{1}$. Equations (9)-(11) determine this location in dimensionless variables.

The amplitude $Q_{1}$ is a constant for every critical state. Ultimately, we have to calculate $Q_{1}$, because, according to (26)-(29), this provides a first approximation of the shapes of the bifurcated equilibrium surfaces. In the process of solving for $Q_{1}$, the region of existence and the stability of the bifurcated states are both determined. 
To obtain $Q_{1}$, the solvability conditions for the boundary-value problems for higher-order approximations are used. Each of the resulting Fredholm-type problems is self-conjugate and has the form

$$
\begin{aligned}
& \left.L N_{i}-q_{i}=f_{i},\right\urcorner N_{i}\left( \pm s_{1}, \varphi\right)=0, \\
& \int_{\Gamma} N_{i} d \Gamma=J_{i} \quad(i \geqslant 2),
\end{aligned}
$$

where $f_{i}$ and $J_{i}$ depend on the shape of $\Gamma$ and on the preceding solutions $\left\{N_{k}, q_{k}\right\}(k<i)$. Since the homogeneous problem (21) has the nontrivial solutions $\left\{N_{1}, q_{1}\right\}$, (34) has solutions if and only if the orthogonality conditions

$$
\left.\left.\int_{\Gamma} N_{1} f_{i} d \Gamma-q_{1} J_{i}=0,\right\urcorner i \geqslant 2,\right\urcorner
$$

are satisfied for each $\left\{N_{1}, q_{1}\right\}$.

The cases for which (21) has a unique nontrivial solution will be analyzed later. The points $C$ and $E$ of the stability boundary, where two linearly independent nontrivial solutions exist [solutions (26), (27) and (27), (28), respectively), will not be considered.

If a change in system parameters results in a transformation from one critical equilibrium state to another, then there is no bifurcation. Therefore, for fixed $\Lambda$, if only the volume is varied, $N_{1}=0$ at the point where the tangent to the stability region boundary is vertical (i.e., at the point $B$ of Fig. 2). For other points on the stability boundary, the approximate solution found by allowing only variations in $V$ enables us to find the approximate solution for arbitrary variations of the system parameters (in particular, when $V$ is fixed and $\Lambda$ changes).

\section{THE BIFURCATION EQUATIONS}

In this section we derive the bifurcation equations. These equations determine the values of $Q_{1}$ for the different segments of the stability boundary and are distinguished by the nature of the critical perturbations that lead to neutral stability. In particular, we emphasize the new results obtained for the stability boundary segment $m A B C$ corresponding to critical nonaxisymmetric perturbations.

\section{A. Segment $m A B C$}

From (A2) (in Appendix A) and (26), we obtain

$$
\begin{aligned}
f_{2}=Q_{1}^{2} F_{0}(s)+Q_{1}^{2} F_{2}(s) \cos 2 \varphi, \\
F_{0}(s)=\frac{1}{4} \frac{z^{\prime 2}}{r^{2}}\left(\beta^{\prime}+3 \frac{z^{\prime}}{r}\right)-F(s), \\
F_{2}(s)=\frac{1}{4} \frac{z^{\prime 2}}{r^{2}}\left(\beta^{\prime}-5 \frac{z^{\prime}}{r}\right)+F(s), \\
F(s)=\frac{1}{2}\left[\beta^{\prime 3}\left(\frac{1}{2} r^{\prime 2}-z^{\prime 2}\right)+\beta^{\prime} \frac{r^{\prime 2} z^{\prime}}{r}\left(4 \frac{z^{\prime}}{r}-\frac{5}{2} \beta^{\prime}\right)\right. \\
\left.+\frac{z^{\prime 5}}{r^{3}}\right],
\end{aligned}
$$

and see that (35) is satisfied for $i=2$.
To obtain the bifurcation equation, we must first find $N_{2}$ and $q_{2}$. The solution, $N_{2}$, of Eq. (22) has the form

$$
\begin{aligned}
N_{2}= & Q_{1}^{2} g_{0}(s)+q_{2} g_{1}(s)+Q_{1}^{2} g_{2}(s) \cos 2 \varphi \\
& +Q_{2} z^{\prime}(s) \sin \varphi,
\end{aligned}
$$

where $Q_{2}$ is constant and $g_{j}(s)(j=0,1,2)$ are the solutions to the equations

$$
L_{0} g_{0}=F_{0}(s), \neg L_{0} g_{1}=1, \neg L_{2} g_{2}=F_{2}(s) .
$$

Since $F_{0}(s)$ and $F_{2}(s)$ are even functions, it then follows both from the symmetry of $\Gamma$ with respect to the equatorial plane and the form of $L_{0}$ and $L_{2}$ that the solution $N_{2}(s, \varphi)$ will satisfy (23) provided that the functions $g_{j}(s)(j$ $=0,1,2)$ are given by

$$
\begin{aligned}
& g_{0}=b_{0}(s)+\alpha_{0} u_{0}(s), \neg g_{1}=u(s)=u_{1}(s)+\alpha_{1} u_{0}(s), \\
& g_{2}=b_{2}(s)+\alpha_{2} u_{2}(s) .
\end{aligned}
$$

Here $u_{0}(s)$ and $u_{1}(s)$ are the solutions of (32) and (33), and $b_{0}(s), b_{2}(s)$ and $u_{2}(s)$ are the solutions to

$$
\begin{aligned}
& \left.L_{0} b_{0}=F_{0}(s) \quad\left(0 \leqslant s \leqslant s_{1}\right), \neg b_{0}(0)=1,\right\urcorner b_{0}^{\prime}(0)=0, \\
& \left.L_{2} b_{2}=F_{2}(s) \quad\left(0 \leqslant s \leqslant s_{1}\right), \neg b_{2}(0)=1,\right\urcorner b_{2}^{\prime}(0)=0, \\
& \left.\left.L_{2} u_{2}=0\right\urcorner \quad\left(0 \leqslant s \leqslant s_{1}\right), \neg \quad u_{2}(0)=1,\right\urcorner u_{2}^{\prime}(0)=0 .
\end{aligned}
$$

The coefficients $\alpha_{0}$ and $\alpha_{2}$ are given by

$$
\alpha_{0}=-b_{0}\left(s_{1}\right) / u_{0}\left(s_{1}\right), \quad \alpha_{2}=-b_{2}\left(s_{1}\right) / u_{2}\left(s_{1}\right) .
$$

Substitution of (26) and (36) into (24), and using the fact that $g_{j}(s)(j=0,1,2)$ are even functions, leads to the equality

$$
\left.q_{2}=Q_{1}^{2} D_{1} \pm D_{2},\right\urcorner
$$

where $D_{1}$ and $D_{2}$ are given by

$$
\begin{aligned}
& D_{1}=\frac{1}{4 G_{1}} q_{0} \int_{0}^{s_{1}} r z^{\prime 2} d s-\frac{G_{0}}{G_{1}}, \\
& D_{2}=\frac{1}{2 G_{1}} r^{2}\left(s_{1}\right)\left|z\left(s_{1}\right)\right|, \neg G_{k}=\int_{0}^{s_{1}} g_{k} r d s \quad(k=0,1) .
\end{aligned}
$$

Finally, from the solvability condition

$$
\int_{\Gamma} N_{1} f_{3} d \Gamma=0
$$

for the third approximation problem, together with the expression for $f_{3}$ [see (A3) in Appendix A] and (26), and (36)(38), we obtain the bifurcation equation

$$
Q_{1}^{2} B_{1} \pm A_{1}=0 . \neg
$$

The values of $A_{1}$ and $B_{1}$ depend on the shape of the profile of the critical equilibrium surface that can be determined according to the method described in Sec. II. Expressions for $A_{1}$ and $B_{1}$ are rather cumbersome, and are given in Appen$\operatorname{dix} \mathrm{B}$.

\section{B. Segment $C D E$}

On the segment $m A B C$ the orthogonality condition (35) is identically satisfied for $i=2$, while the bifurcation equation is deduced from the condition (35) for $i=3$. A similar 
situation arises in the analysis of bifurcation along $E F n$. This is due to the nature of the problem where the critical axisymmetric surface (with an equatorial symmetry plane) is neutrally stable either to nonaxisymmetric perturbations (segment $m A B C$ ) or to an axisymmetric perturbation that is antisymmetric with respect to the equatorial plane (segment $E F n$ ). Along $C D E$, the critical perturbation is axisymmetric and equatorially symmetric. Here the bifurcation equation is deduced from the solvability condition (35) for the second approximation problem $(i=2)$. It has the form

$$
Q_{1}^{2} B_{2}=-1 . \neg
$$

The expression for the coefficient $B_{2}$ is presented in Appendix B.

\section{Segment EFn}

For all points of this segment, except the point $F$, the equalities (28) hold. Using the first of them, we obtain $f_{2}$ $=Q_{1}^{2} F_{3}(s)$ and

$$
F_{3}=r^{\prime 2}\left(\beta^{\prime}-\frac{z^{\prime}}{r}\right)^{3}-\frac{1}{2} z^{\prime 2} \beta^{\prime 2}\left(\beta^{\prime}-\frac{z^{\prime}}{r}\right)+q_{0} \beta^{\prime} \frac{z^{\prime}}{r} r^{\prime 2} .
$$

It can be readily verified that (35) is satisfied for $i=2$. Upon determination of $N_{2}$ and $q_{2}$, the condition (35) for $i=3$ reduces to the following bifurcation equation:

$$
\left.Q_{1}^{2} B_{3} \pm A_{3}=0 .\right\urcorner
$$

The coefficients $A_{3}$ and $B_{3}$ are presented in Appendix B.

\section{NUMERICAL AND ANALYTICAL RESULTS}

In this section we present the results of numerical and analytical analyses of the bifurcation equations obtained in Sec. V. In particular, Secs. VI A and B deal with bifurcations to nonaxisymmetric shapes and Table II summarizes the types of bifurcation along each boundary segment. Except for the case of catenoid, we use dimensionless quantities with $q_{0}^{-1}$ as the characteristic length. For cross reference with dimensional equations, we note that all dimensional quantities except $q_{0}$ are replaced in each equation by their dimensionless analogs, while $q_{0}$ is set equal to unity. All dimensionless quantities, except $\rho, \zeta$, and $\tau$ [see (3)], are distinguished from their dimensional counterparts by a bar overscript.

\section{A. Segment Am}

Numerical results for the $A T_{1}$ segment $[\Lambda<0.4946$, Fig. 2(a)] show that $\bar{A}_{1}>0$ and $\bar{B}_{1}<0$. In contrast, within the $T_{1} m$ branch $(\Lambda>0.4946)$ both $\bar{A}_{1}$ and $\bar{B}_{1}$ are positive. The dependence of $\overline{A_{1}} / \bar{B}_{1}$ on $\Lambda$ is shown in Fig. 4.

Taking (7) into account, expansions in powers of the small parameter $\nu=\rho_{0}^{-1}$ can be used to obtain asymptotic expressions for $\rho(\tau), \zeta^{\prime}(\tau), \tau_{1}, \overline{u_{0}}(\tau), \overline{u_{1}}(\tau), \overline{u_{2}}(\tau), \overline{b_{0}}(\tau)$, $\bar{b}_{2}(\tau), \Lambda, \bar{A}_{1}$, and $\bar{B}_{1}$ at large values of $\rho_{0}$. Restricting ourselves to the determination of the two principal terms which define the behavior of $\bar{A}_{1}$ and $\bar{B}_{1}$, we arrive at the equalities

$$
\Lambda=\nu+\nu^{2}(1+\pi / 4)+o\left(\nu^{2}\right)
$$

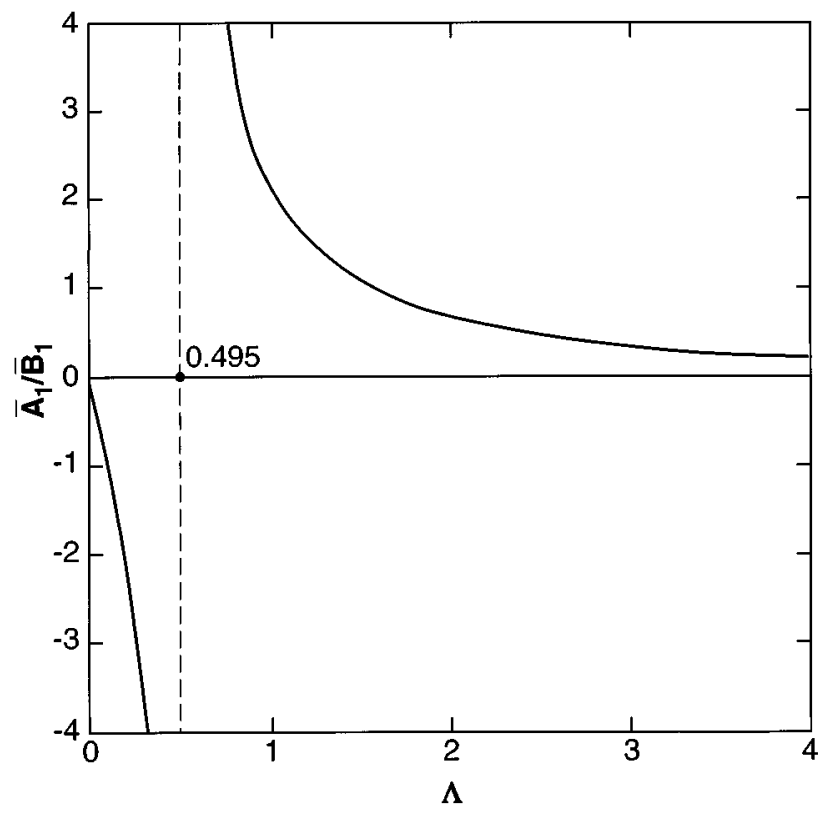

FIG. 4. The ratio of the bifurcation equation coefficients, $\bar{A}_{1} / \bar{B}_{1}$, as a function of slenderness, $\Lambda$, within the segment $A m$ of the stability boundary.

$$
\begin{aligned}
& \overline{A_{1}}=\frac{1}{2 \nu^{2}}\left[1-\nu\left(2+\frac{\pi}{4}\right)\right]+o\left(\nu^{-1}\right), \neg \\
& \bar{B}_{1}=-\frac{1}{6 \pi \nu^{3}}(1-3 \nu)+o\left(\nu^{-2}\right),
\end{aligned}
$$

from which we can see that

$$
\begin{aligned}
\left(\bar{Q}_{1}\right)_{1} & =-\left(\bar{Q}_{1}\right)_{2} \\
& \left.=\left|\bar{A}_{1} / \bar{B}_{1}\right|^{1 / 2}=\sqrt{3 \pi \Lambda}\left(1-\frac{\pi}{4} \Lambda\right)+o\left(\Lambda^{3 / 2}\right) .\right\urcorner
\end{aligned}
$$

Equations (42) and (43) are in full agreement with numerical results for small $\Lambda$ (or large $\rho_{0}$ ).

The coefficient $\bar{B}_{1}$ vanishes at the point $T_{1}$ (see Table I). It should also be noted that the radius of convergence of the expansions (19) tends to zero as the point $T_{1}$ is approached. This means that in a small neighborhood of the point $T_{1}$, the shapes of bifurcated surfaces cannot be expressed as a series in half-integral powers of $\left|V-V_{*}\right|$. In this case, it should be expected that expansions in powers $\left|V-V_{*}\right|^{1 / 3}$ are valid. ${ }^{10}$

Inspection of the signs of $\overline{A_{1}}$ and $\bar{B}_{1}$ and of the direction of motion along critical surface profiles (see Sec. III) reveals that real solutions of the dimensionless form of (39) exist in a neighborhood $\left(\left|V-V_{*}\right| \ll 1\right)$ either along $A T_{1}$ for $V<V_{*}$, or along $T_{1} m$ for $V>V_{*}$. To a first approximation, these solutions determine (to within an arbitrary rotation about the $\zeta$ axis) the shape perturbation of surfaces that bifurcate in the indicated $\left(V<V_{*}\right.$ or $\left.V>V_{*}\right)$ directions: $\bar{N}=\varepsilon \sqrt{\left|\bar{A}_{1} / \bar{B}_{1}\right|} \zeta^{\prime}(\tau) \sin \varphi$.

On the boundary $A m$, the axisymmetric equilibrium state is critical with respect to nonaxisymmetric perturbations and is stable with respect to axisymmetric ones. The problem (21) has a nonaxisymmetric solution and has no nontrivial 


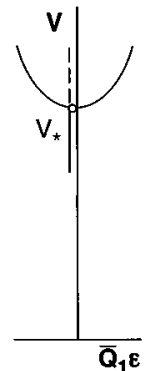

(a)

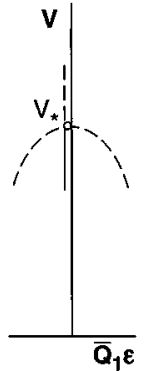

(b)

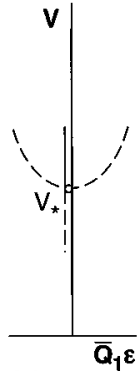

(c)

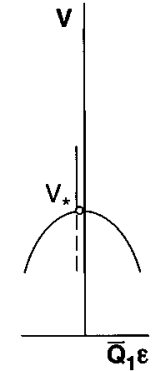

(d)

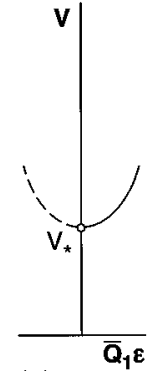

(e)
FIG. 5. Bifurcation diagrams in the plane of the dimensionless variables $\bar{Q}_{1} \epsilon$ and $V$ for the critical axisymmetric states belonging to segments (a) $T_{1} m$ and $B T_{3}$, (b) $A T_{1}$ and $T_{3} C$, (c) $A T_{2}$ and $E F n$, (d) $T_{2} B$, and (e) $C D E$ of the stability boundary. The stable states are denoted by solid curves $(-)$ and unstable ones by dashed curves (---). The thick vertical solid line $\bar{Q}_{1} \varepsilon$ $=0$ corresponds to axisymmetric shapes that have an equatorial symmetry plane (for emphasis, this line is separated from the $V$ axis).

axisymmetric solutions. Thus, axisymmetric solutions of the equilibrium problem have a unique continuous extension into the supercritical region and can be expressed as a series in integral powers of $\left(V-V_{*}\right)$ provided that this quantity is small. Sketches of the bifurcations at points within the segments $T_{1} m$ and $A T_{1}$ are shown in Figs. 5(a) and 5(b), respectively.

For $\Lambda>0.4946$, a bridge will adopt stable nonaxisymmetric shapes whenever stability is lost on the upper boundary. Plateau ${ }^{1}$ observed such continuous shape transitions in experiments more than a century ago. For $\Lambda<0.4946$, however, an identical evolution leads to a discontinuity. According to our experimental results (see Sec. VII), the system undergoes a finite jump from a critical state to a stable nonaxisymmetric one.

Fowle et $a l .^{29}$ (experimentally) and Brown and Scriven ${ }^{20}$ (theoretically) reached the same qualitative conclusion for rotating weightless bridges. They found that for critical "thick" $(V \gg 1)$ bridges, a supercritical bifurcation to stable families of nonaxisymmetric shapes was possible. Alternatively, for slightly bulged bridges (i.e., bridges with $V$ slightly more than 1), loss of stability to nonaxisymmetric perturbations leads to subcritical bifurcation. ${ }^{20}$

\section{B. Segment $A B C$}

According to our numerical results, $\overline{A_{1}}$ is positive along $A B$, changes its sign at the point $B$, and is negative along $B C$. The sign change at point $B$ has been explained at the end of Sec. IV. The coefficient $\bar{B}_{1}$ has negative values within the segments $A T_{2}$ and $T_{3} C$ and is positive within the segment $T_{2} B T_{3}$ [Fig. 2(b)]. The points $T_{2}$ and $T_{3}$ (see Table I), like the point $T_{1}$, are singular. The numerical values of $\bar{A}_{1} / \bar{B}_{1}$ along the segment $A B C$ are represented in Fig. 6.

From (8), we can deduce expansions similar to those described in Sec. VI A. We then obtain the principal terms that define the behavior of $\Lambda, \bar{A}_{1}$, and $\bar{B}_{1}$ for large values of $\rho_{0}$ (i.e., near the point $A$ ). Finally, in place of (42) and (43) we have
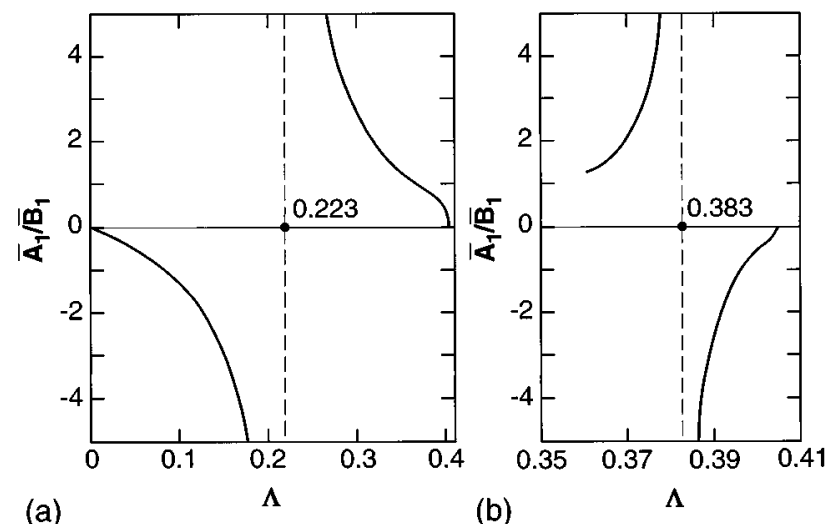

FIG. 6. Dependence of coefficients ratio $\bar{A}_{1} / \bar{B}_{1}$ on $\Lambda$ within the segments $A T_{2} B$ (a) and $C T_{3} B$ (b) of the stability boundary.

$$
\begin{aligned}
& \Lambda=\nu-\nu^{2}(1+\pi / 4)+o\left(\nu^{2}\right) \\
& \bar{A}_{1}=\frac{1}{2 \nu^{2}}\left[1+\nu\left(2+\frac{\pi}{4}\right)\right]+o\left(\nu^{-1}\right), \\
& \bar{B}_{1}=-\frac{1}{6 \pi \nu^{3}}(1-3 \nu)+o\left(\nu^{-2}\right),
\end{aligned}
$$

and

$$
\left(\bar{Q}_{1}\right)_{1}=-\left(\bar{Q}_{1}\right)_{2}=\sqrt{3 \pi \Lambda}\left(1+\frac{\pi}{4} \Lambda\right)+o\left(\Lambda^{3 / 2}\right) .
$$

From the signs of the coefficients $\bar{A}_{1}$ and $\bar{B}_{1}$, and from the direction of motion along the critical profiles, it can be concluded that the bifurcation takes place into the region $V$ $>V_{*}$ within the segments $A T_{2}$ and $B T_{3}$ and into the region $V<V_{*}$ within the segments $T_{2} B$ and $T_{3} C$. Consequently, the bifurcation is supercritical within $T_{2} B T_{3}$, and is subcritical within $A T_{2}$ and $T_{3} C$ [Figs. 5(a)-5(d)].

\section{Segments $C D E$ and $E F n$ and special cases}

A detailed analysis of the bifurcation among axisymmetric bridge shapes has been performed by Lowry and Steen. ${ }^{3}$ In particular, for fixed values of $\Lambda$, they constructed families of possible axisymmetric equilibrium states and presented them in the $(p, V)$-plane ( $p=a q$ is the dimensionless pressure jump at a free surface). The $(p, V)$-diagrams so obtained represent the bifurcation structure. From these diagrams, the stability or instability to axisymmetric perturbations can also be determined for different equilibrium branches (or parts of them) using the location of the turning points in volume and the branch points.

It has also been shown ${ }^{3}$ that interior points of the boundary segment $C D E$ are the turning points in volume for axisymmetric equatorially symmetric states. Thus, two branches of axisymmetric equatorially symmetric states that are located in the subcritical region $V>V_{*}$ bifurcate from a critical state corresponding to a point within $C D E$. One of the branches consists of stable states, and the other of unstable ones [Fig. 5(e)]. The unstable axisymmetric (but equatorially 
antisymmetric) states bifurcate from every interior point of $E F n$ into the subcritical region $V>V_{*}$ [Fig 5(c)].

Results of our calculations of the coefficients $B_{2}, A_{3}$, and $B_{3}$ of the bifurcation equations (40) and (41) along $C D E$ and $E F n$ are in complete agreement with the above conclusions. Thus, we present results only for special cases.

Critical catenoid. The parameters of the critical catenoid (the point $D$ ) are given in Table I. In addition, we have $s_{1}$ $=-r_{0} / \tan \beta_{1}=4.6396 r_{0}$, where $r_{0}$ is the equatorial radius of the critical catenoid $\left(r_{0}=a \sin \beta_{1}=0.2107 a\right)$. We found that $B_{2}=-3.5347 r_{0}^{2}$ for the case when the domain $\Omega$ is to the left with increasing $s$. Thus, for $V>V_{*}=0.2405$, there are two branches of equilibrium surfaces that bifurcate from the critical catenoid. They are characterized by $N_{1}$ $= \pm 0.5319 u r_{0}^{-1}$. The dimensionless function $u(s) r_{0}^{-2}$ for the critical catenoid was calculated using (31)-(33).

Almost cylindrical critical surfaces. The solution of the bifurcation problem for the critical surfaces close to that of a cylinder is found using (7) and expansions for $\rho(\tau), \zeta^{\prime}(\tau), \Lambda$, $\bar{u}_{0}(\tau), \bar{u}_{1}(\tau)$, and $\bar{b}_{3}(\tau)$ (see Appendix B) in terms of the small parameter $\mu_{1}=\rho_{0}-1$. This yields the following result

$$
\left.\bar{Q}_{1}= \pm \sqrt{\frac{2}{3}} \frac{(2 \pi-\Lambda)}{|\pi-\Lambda|}+o(1) .\right\urcorner
$$

The discrepancy between the values obtained from (44) and the numerical results is within $1 \%$ for $|\pi-\Lambda| \leqslant 0.35$. Furthermore, since $\bar{N}_{1}=\bar{Q}_{1} \rho^{\prime}(\tau)$, we find that

$$
\begin{aligned}
\bar{N}_{1}= & \pm \sqrt{\frac{2}{3}}\left[1+\left(1-\frac{\Lambda}{\pi}\right)\left(\frac{7}{4}+\cos \tau\right)\right] \\
& \times \sin \tau+o\left(\left|1-\frac{\Lambda}{\pi}\right|\right) \cdot \neg
\end{aligned}
$$

The result for a critical cylinder $(\Lambda=\pi)$ is a special case of (45).

From the general theory of bifurcation of solutions of nonlinear equations (see, for example, Ref. 30), it can be proved that if the coefficients $\overline{A_{i}}$ and $\overline{B_{i}}$ of the bifurcation equation are nonzero, then there are only two branches of bifurcated equilibrium shapes. Furthermore, these shapes can be represented as a series in half-integral powers of the deviation of the equilibrium parameters from their critical values. This justifies our earlier assumption that the rough type of bifurcation can be used at all points of the stability boundary except at the singular points $T_{1}, T_{2}$, and $T_{3}$ (and the points $C$ and $E$ which were not examined here).

\section{EXPERIMENTS}

In Sec. VI A we described the nature of the stability boundary segment $\mathrm{Am}$. Here the axisymmetric equilibrium state is critical with respect to nonaxisymmetric perturbations and is stable to axisymmetric ones. We noted that on this segment with $\Lambda>\Lambda_{s}=0.4946$, a bridge will continuously adopt stable nonaxisymmetric shapes upon loss of stability of axisymmetric shapes. However, for $\Lambda<\Lambda_{s}$, we predicted that loss of stability leads to a discontinuous change of a critical shape.
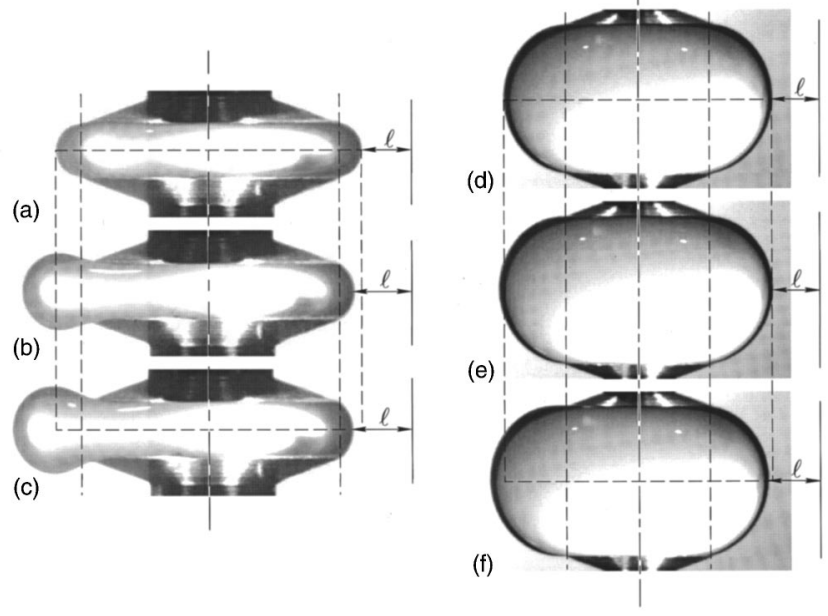

FIG. 7. Bridge images for $\Lambda=0.225[(\mathrm{a})-(\mathrm{c})]$ and $\Lambda=1.02[(\mathrm{~d})-(\mathrm{f})]$. States (a) and (d) are stable axisymmetric ones with volumes $V_{1}=1.31$ and $V_{2}$ $=2.82$ that are, respectively, slightly less the associated (experimental) critical volumes of 1.33 and 2.83 for the given $\Lambda$. The nonaxisymmetric bridges (b) and (c) have volumes of $V_{1}+0.025$ and $V_{1}+0.05$, while (e) and (f) have volumes of $V_{2}+0.095$ and $V_{2}+0.19$.

To investigate this we performed experiments in a 3.8 $\times 10^{4} \mathrm{~cm}^{3}$ Plateau tank under almost neutrally buoyant conditions. A silicone oil (5 cs. polydimethylsiloxane Dow Corning 200 series) was used as a bridge liquid, and a mixture of water and methanol as a surrounding liquid bath. The mixture composition was adjusted to achieve a density difference, $\Delta \rho$, between mixture and oil as small as possible. Densities were measured accurately to $2.5 \times 10^{-4} \mathrm{~g} / \mathrm{cm}^{3}$ using a calibrated hydrometer. The estimated maximum value of $\Delta \rho$ was $5 \times 10^{-4} \mathrm{~g} / \mathrm{cm}^{3}$. The interfacial tension, $\sigma$, was $14.5 \pm 0.5$ dyne $/ \mathrm{cm}$. The disk radius $a$ was $0.5 \pm 0.002 \mathrm{~cm}$. Experiments were conducted in a temperature-controlled room which, together with the large thermal mass of the bath, led to small temperature variations in the tank. Recorded temperature variations did not exceed $0.01 \mathrm{~K}$ over 30 min, so $\sigma$ and $\Delta \rho$ were almost constant for the duration of each experiment. Thus, the magnitude of the Bond number, $B=\Delta \rho g a^{2} / \sigma$ (here $g=980 \mathrm{~cm} / \mathrm{s}^{2}$ ), was at most $10^{-2}$.

A liquid bridge of a set slenderness was formed between coaxial disks. High precision stepping motors were used to control the disk separation while simultaneously injecting silicone oil. Oil was injected until the bridge was near the upper stability limit for the slenderness under consideration. Precisely controlled amounts of oil were then added incrementally using a calibrated microsyringe. The relative volume increment was about 1 part in 200. After each addition of oil, the bridge was allowed to deform and come to rest. The bridge was imaged using a coherent high-magnification Fourier optical arrangement together with a high-pass optical filter. This permits visualization of edges of projections of the liquid bridge surface at approximately $100 \times$ magnification. From this image, the minimum distance $l$ (shown schematically on the larger scale photographs in Fig. 7) from the bridge surface to a stationary reticule was measured on a computer screen. Typically, the distance decreases slightly with each addition of oil until the stability limit is exceeded. 
When this occurs, the bridge forms a bulge. The corresponding deformation of the bridge is characterized as follows. First the projection of the edge of the surface $180^{\circ}$ from the maximum bulge is located. The distance between the edge of the projected image of the bridge surface opposite the bulge and the stationary reticule is then recorded. Further incremental additions of oil lead to an increase in the distance between the edge of the image and the reticule. This distance is recorded for each increment. Because the resulting incremental deformations of the bridge are on the order of several microns, care was taken to ensure the bridge was properly isolated from vibration. It was also necessary to ensure that there was no backlash in the injection system.

A similar experimental technique was used by Russo and Steen ${ }^{17}$ except that they fixed the actual bridge volume, $v$, and decreased the disk separation to find the maximum relative volume stability limit. As regards this limit for axisymmetric states, our experiments (like the experiments ${ }^{17}$ performed for $\Lambda \leqslant 1$ ) agreed with the theoretical prediction (segment $A m$ ) for relatively small values of $\Lambda$. However, at large $\Lambda$, tangents at the endpoints of critical surface profiles showed a discernible deviation from the horizontal. Here, the critical axisymmetric states were found to occur below the theoretical limit. With larger values of $\Lambda$ (and volumes), an axisymmetric bridge is more sensitive to real finite amplitude disturbances and to nonzero Bond numbers.

After loss of stability, the critical axisymmetric shape changes to a stable nonaxisymmetric shape. However, the nature of this transition was found to be quite different for $\Lambda \leqslant 0.4$ and $\Lambda \geqslant 0.6$. Figure 7 shows a sequence of images of a $\Lambda=0.225$ bridge near the critical $V$ value. The theoretically predicted critical volume is 1.36 , the critical volume obtained experimentally was 1.33 . For $\Lambda=0.225$, a large shape deformation occurs after the addition of only a small volume increment [compare Figs. 7(a) and 7(b)]. Further volume increases lead to continuous incremental shape changes [Figs. 7(b) and 7(c)]. In contrast, for $\Lambda=1.02$ [Figs. 7 (d) -7 (f)], we observed a continuous transition from critical axisymmetric shape to a sequence of the stable nonaxisymmetric shapes as the volume was increased. Here the theoretical critical volume is 2.96 and our experimental critical volume was between 2.83 and 2.85. In both cases, our experimental critical volumes were within $5 \%$ of the theoretical values and the nonaxisymmetric bridges were stable at volumes far beyond the maximum volume stability limit for axisymmetric bridges.

The graphs in Fig. 8 show the rate of change of dimensionless deformation $\xi=l / a$ with the relative volume in the vicinity of the axisymmetric to nonaxisymmetric transition. For $\Lambda \leqslant 0.4$, the curve peaks sharply in the immediate vicinity of the critical volume. With smaller $\Lambda$, the maximum value of $d \xi / d V$ becomes larger. In contrast, for $\Lambda \geqslant 0.6$, the curve is rounded, and deformation occurs continuously over a much larger range of volume. Note that even for $\Lambda=0.40$, the maximum value of $d \xi / d V$ is much larger than for $\Lambda=0.60$.

Although hysteresis was expected for $\Lambda<\Lambda_{s}$, no hysteresis was observed either in our experiments or in those of Russo and Steen. ${ }^{17}$ It is possible that the unstable bifurcating

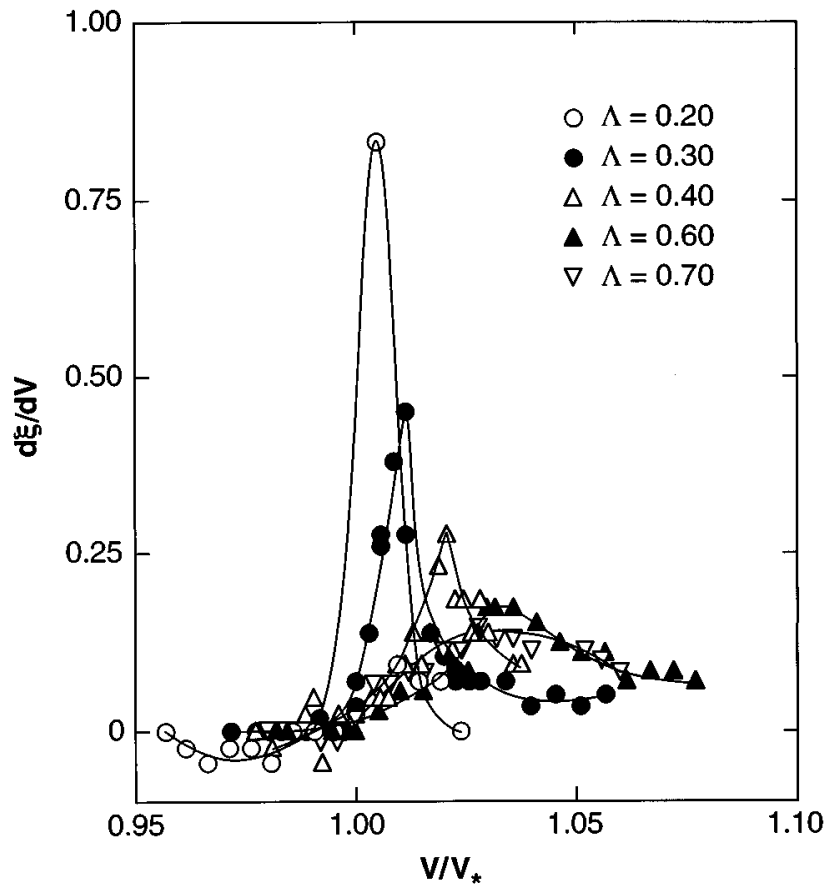

FIG. 8. Rate of change of dimensionless bridge deformation $\xi$ with relative volume $(d \xi / d V)$ as a function of relative volume $V$ in the vicinity of the critical volume $V_{*}$ corresponding to the axisymmetric-nonaxisymmetric transition.

nonaxisymmetric states exist only in a small subcritical vicinity of the segment $A T_{1}$.

\section{CONCLUSIONS}

To a first approximation, the bifurcation problem has been solved for all axisymmetric critical states of a liquid bridge except those corresponding to the points $T_{1}, T_{2}$, $T_{3}, C$, and $E$ on the boundary of the stability region shown in Fig. 2. Motivated by the lack of a comprehensive systematic study of states that are critical to nonaxisymmetric perturbations, particular emphasis has been placed on the examination of this aspect of the bifurcation problem. States that are critical to nonaxisymmetric perturbations lie on the segment $m A B C$ of the stability boundary for axisymmetric bridges. This segment contains the singular points $T_{1}, T_{2}$, and $T_{3}$. We found that if the path of the stability region crosses the $T_{1} m$ or the $T_{2} B T_{3}$ segments, stable nonaxisymmetric liquid bridges appear. In this case, the stable axisymmetric states are replaced continuously by the stable nonaxisymmetric ones. Expressions for these nonaxisymmetric shapes near the stability boundary were obtained. When stability is lost at the boundary segment $T_{1} A T_{2}$ or $T_{3} C$, the bridge shape changes discontinuously. In particular, loss of stability along $T_{1} A$ was seen to occur through a large deformation from the critical axisymmetric state to a stable nonaxisymmetric state.

\section{ACKNOWLEDGMENTS}

This work was supported by the National Aeronautics and Space Administration through grant NAG3-1384, by IN- 
TAS (project number INTAS-94-0529), and by the State of Alabama through the Alabama Supercomputer Network. The basic results were discussed with Dr. A. D. Tyuptsov just before his sudden death. The authors are sincerely thankful to Drs. J. M. Perales and M. A. Svechkareva for help with the calculations.

\section{APPENDIX A: THE MEAN CURVATURE $\boldsymbol{H}$ OF A PERTURBED SURFACE AND THE FUNCTIONS $\boldsymbol{f}_{2}$ AND $\boldsymbol{f}_{3}$}

$$
\begin{aligned}
& 2 H=\left(\beta^{\prime}+\frac{z^{\prime}}{r}\right)+\left(\beta^{\prime 2}+\frac{z^{\prime 2}}{r^{2}}\right) N+\frac{r^{\prime}}{r} N_{s}^{\prime}+N_{s}^{\prime \prime}+\frac{1}{r^{2}} N_{\varphi \varphi}^{\prime \prime}+\left(\beta^{\prime 3}+\frac{z^{\prime 3}}{r^{3}}\right) N^{2}+\left(\frac{r^{\prime} z^{\prime}}{r^{2}}+\frac{r^{\prime}}{r} \beta^{\prime}+\beta^{\prime \prime}\right) N N_{s}^{\prime} \\
& +\frac{1}{2}\left(\beta^{\prime}-\frac{z^{\prime}}{r}\right) N_{s}^{\prime 2}-\frac{1}{2 r^{2}}\left(\beta^{\prime}-\frac{z^{\prime}}{r}\right) N_{\varphi}^{\prime 2}+2 \beta^{\prime} N N_{s s}^{\prime \prime}+\frac{2 z^{\prime}}{r^{3}} N N_{\varphi \varphi}^{\prime \prime}+\left(\beta^{\prime 4}+\frac{z^{\prime 4}}{r^{4}}\right) N^{3} \\
& +\left[\frac{r^{\prime}}{r}\left(\beta^{\prime 2}+\frac{z^{\prime}}{r} \beta^{\prime}+\frac{z^{\prime 2}}{r^{2}}\right)+3 \beta^{\prime} \beta^{\prime \prime}\right] N^{2} N_{s}^{\prime}+\frac{1}{2}\left(3 \beta^{\prime 2}-2 \frac{z^{\prime}}{r} \beta^{\prime}-\frac{z^{\prime 2}}{r^{2}}\right) N N_{s}^{\prime 2}-\frac{1}{2} \frac{r^{\prime}}{r} N_{s}^{\prime 3} \\
& -\frac{1}{2 r^{2}}\left(\beta^{\prime 2}+2 \frac{z^{\prime}}{r} \beta^{\prime}-3 \frac{z^{\prime 2}}{r^{2}}\right) N N_{\varphi}^{\prime 2}+\frac{1}{2} \frac{r^{\prime}}{r^{3}} N_{s}^{\prime} N_{\varphi}^{\prime 2}+3 \beta^{\prime 2} N^{2} N_{s s}^{\prime \prime}-\frac{3}{2} N_{s}^{\prime 2} N_{s s}^{\prime \prime}-\frac{1}{2 r^{2}} N_{\varphi}^{\prime 2} N_{s s}^{\prime \prime}+\frac{3 z^{\prime 2}}{r^{4}} N^{2} N_{\varphi \varphi}^{\prime \prime} \\
& \left.-\frac{1}{2 r^{2}} N_{s}^{\prime 2} N_{\varphi \varphi}^{\prime \prime}-\frac{3}{2 r^{4}} N_{\varphi}^{\prime 2} N_{\varphi \varphi}^{\prime \prime}-\frac{2}{r^{2}} N_{s}^{\prime} N_{\varphi}^{\prime} N_{s \varphi}^{\prime \prime}+o\left(N^{3}\right) ;\right\urcorner \\
& \left.f_{2}=-\left(\beta^{\prime 3}+\frac{z^{\prime 3}}{r^{3}}\right) N_{1}^{2}-2 \frac{r^{\prime} z^{\prime}}{r^{2}} N_{1} N_{1 s}^{\prime}-\frac{1}{2}\left(\beta^{\prime}-\frac{z^{\prime}}{r}\right) N_{1 s}^{\prime 2}-2 \beta^{\prime} N_{1} N_{1 s s}^{\prime \prime}+\frac{1}{2 r^{2}}\left(\beta^{\prime}-\frac{z^{\prime}}{r}\right) N_{1 \varphi}^{\prime 2}-\frac{2 z^{\prime}}{r^{3}} N_{1} N_{1 \varphi \varphi}^{\prime \prime} ;\right\urcorner \\
& f_{3}=-2\left(\beta^{\prime 3}+\frac{z^{\prime 3}}{r^{3}}\right) N_{1} N_{2}-2 \frac{r^{\prime} z^{\prime}}{r^{2}}\left(N_{1} N_{2 s}^{\prime}+N_{2} N_{1 s}^{\prime}\right)-\left(\beta^{\prime}-\frac{z^{\prime}}{r}\right) N_{1 s}^{\prime} N_{2 s}^{\prime}-2 \beta^{\prime}\left(N_{1} N_{2 s s}^{\prime \prime}+N_{2} N_{1 s s}^{\prime \prime}\right) \\
& +\frac{1}{r^{2}}\left(\beta^{\prime}-\frac{z^{\prime}}{r}\right) N_{1 \varphi}^{\prime} N_{2 \varphi}^{\prime}-\frac{2 z^{\prime}}{r^{3}}\left(N_{1} N_{2 \varphi \varphi}^{\prime \prime}+N_{2} N_{1 \varphi \varphi}^{\prime \prime}\right)-\left(\beta^{\prime 4}+\frac{z^{\prime 4}}{r^{4}}\right) N_{1}^{3}-\frac{r^{\prime}}{r}\left(\frac{z^{\prime 2}}{r^{2}}+4 \frac{z^{\prime}}{r} \beta^{\prime}-2 \beta^{\prime 2}\right) \\
& \times N_{1}^{2} N_{1 s}^{\prime}-\frac{1}{2}\left(3 \beta^{\prime 2}-2 \frac{z^{\prime}}{r} \beta^{\prime}-\frac{z^{\prime 2}}{r^{2}}\right) N_{1} N_{1 s}^{\prime 2}+\frac{1}{2} \frac{r^{\prime}}{r} N_{1 s}^{\prime 3}+\frac{1}{2 r^{2}}\left(\beta^{\prime 2}+2 \frac{z^{\prime}}{r} \beta^{\prime}-3 \frac{z^{\prime 2}}{r^{2}}\right) N_{1} N_{1 \varphi}^{\prime 2} \\
& -\frac{1}{2} \frac{r^{\prime}}{r^{3}} N_{1 s}^{\prime} N_{1 \varphi}^{\prime 2}-3 \beta^{\prime 2} N_{1}^{2} N_{1 s s}^{\prime \prime}+\frac{3}{2} N_{1 s}^{\prime 2} N_{1 s s}^{\prime \prime}+\frac{1}{2 r^{2}} N_{1 \varphi}^{\prime 2} N_{1 s s}^{\prime \prime}-\frac{3 z^{\prime 2}}{r^{4}} N_{1}^{2} N_{1 \varphi \varphi}^{\prime \prime}+\frac{1}{2 r^{2}} N_{1 s}^{\prime 2} N_{1 \varphi \varphi}^{\prime \prime} \\
& +\frac{3}{2 r^{4}} N_{1 \varphi}^{\prime 2} N_{1 \varphi \varphi}^{\prime \prime}+\frac{2}{r^{2}} N_{1 s}^{\prime} N_{1 \varphi}^{\prime} N_{1 s \varphi}^{\prime \prime} \cdot \neg
\end{aligned}
$$

Here, the primes denote partial derivatives of the functions $N, N_{1}$, and $N_{2}$ with respect to the arguments indicated in the subscripts.

\section{APPENDIX B: COEFFICIENTS $A_{1}, B_{1}, B_{2}, A_{3}$, AND $B_{3}$ OF THE BIFURCATION EQUATIONS}

The coefficients $A_{1}$ and $B_{1}$ of the equation (39) are

$$
\begin{aligned}
& \left.A_{1}=\frac{1}{G_{1}} r^{2}\left(s_{1}\right)\left|z\left(s_{1}\right)\right| E_{1},\right\urcorner G_{1}=\int_{0}^{s_{1}} u_{1} r d s+\alpha_{1} \int_{0}^{s_{1}} u_{0} r d s, \\
& E_{1}=\int_{0}^{s_{1}}\left(M_{11} u_{1}+M_{12} u_{1}^{\prime}+M_{13} u_{1}^{\prime \prime}\right) d s+\alpha_{1} \int_{0}^{s_{1}}\left(M_{11} u_{0}+M_{12} u_{0}^{\prime}+M_{13} u_{0}^{\prime \prime}\right) d s,
\end{aligned}
$$

and

$$
\begin{aligned}
B_{1}= & \int_{0}^{s_{1}}\left[2 M_{11}\left(b_{0}-\frac{1}{2} b_{2}\right)+M_{14}\left(b_{0}^{\prime}-\frac{1}{2} b_{2}^{\prime}\right)+2 M_{13}\left(b_{0}^{\prime \prime}-\frac{1}{2} b_{2}^{\prime \prime}\right)+M_{15} b_{2}+M_{16}\right] d s \\
& +\alpha_{0} \int_{0}^{s_{1}}\left(2 M_{11} u_{0}+M_{14} u_{0}^{\prime}+2 M_{13} u_{0}^{\prime \prime}\right) d s-\frac{1}{2} \alpha_{2} \int_{0}^{s_{1}}\left[2\left(M_{11}-M_{15}\right) u_{2}+M_{14} u_{2}^{\prime}+2 M_{13} u_{2}^{\prime \prime}\right] d s \\
& -\frac{2 E_{1}}{G_{1}}\left[\int_{0}^{s_{1}} b_{0} r d s+\alpha_{0} \int_{0}^{s_{1}} u_{0} r d s-\frac{1}{4} q_{0} \int_{0}^{s_{1}} r z^{\prime 2} d s\right],
\end{aligned}
$$


with

$$
\begin{aligned}
M_{11}= & r^{\prime 2} z^{\prime}\left(\beta^{\prime}-\frac{z^{\prime}}{r}\right)^{2}, \neg M_{12}=-\frac{r^{\prime} z^{\prime}}{r}\left[z^{\prime 2}+\frac{1}{2} r^{2} \beta^{\prime}\left(\beta^{\prime}-\frac{z^{\prime}}{r}\right)\right], \neg M_{13}=-r z^{\prime 2} \beta^{\prime}, \\
M_{14}= & -r r^{\prime} z^{\prime}\left[\left(\beta^{\prime}-\frac{z^{\prime}}{r}\right)^{2}+q_{0} \frac{z^{\prime}}{r}\right], \neg M_{15}=-\frac{z^{\prime 2}}{r}\left(\beta^{\prime}+3 \frac{z^{\prime}}{r}\right), \\
M_{16}= & -r^{\prime 2} z^{\prime}\left(\beta^{\prime}-\frac{z^{\prime}}{r}\right)\left[\frac{9}{8} \beta^{\prime 2}\left(r^{\prime 2}-4 z^{\prime 2}\right)+\frac{z^{\prime 2}}{8 r^{2}}\right]+z^{\prime 4}\left[\frac{3}{2} r \beta^{\prime 4}-\frac{3}{8 r^{3}}\left(1+2 z^{\prime 4}\right)+\frac{z^{\prime}}{8 r^{2}}\left(2 \beta^{\prime}+15 \frac{z^{\prime}}{r}\right)\right] \\
& -\frac{3}{4} r^{\prime 2} z^{\prime 2} \beta^{\prime}\left[3 r \beta^{\prime 3}+\frac{z^{\prime 2}}{r}\left(\frac{1}{2} \beta^{\prime}+\frac{z^{\prime}}{r}\right)-\frac{1}{6 r}\left(\beta^{\prime}-\frac{z^{\prime}}{r}\right)\right]+\frac{3}{8} r^{\prime 4} z^{\prime} \beta^{\prime 3} .
\end{aligned}
$$

The coefficient $B_{2}$ of the equation (40) is

$$
\begin{aligned}
B_{2}= & \frac{2}{r^{2}\left(s_{1}\right)\left|z\left(s_{1}\right)\right|}\left\{\alpha_{1}^{3} \int_{0}^{s_{1}}\left(M_{21} u_{0}^{3}+M_{22} u_{0}^{2} u_{0}^{\prime}+M_{23} u_{0} u_{0}^{\prime 2}+M_{24} u_{0}^{2} u_{0}^{\prime \prime}\right) d s+\alpha_{1}^{2} \int_{0}^{s_{1}}\left[3 M_{21} u_{0}^{2} u_{1}\right.\right. \\
& \left.+M_{22}\left(u_{0}^{2} u_{1}^{\prime}+2 u_{0} u_{0}^{\prime} u_{1}\right)+M_{23}\left(u_{0}^{\prime 2} u_{1}+2 u_{0} u_{0}^{\prime} u_{1}^{\prime}\right)+M_{24}\left(u_{0}^{2} u_{1}^{\prime \prime}+2 u_{0} u_{0}^{\prime \prime} u_{1}\right)+M_{25} u_{0}^{2}\right] d s \\
& +\alpha_{1} \int_{0}^{s_{1}}\left[3 M_{21} u_{0} u_{1}^{2}+M_{22}\left(u_{0}^{\prime} u_{1}^{2}+2 u_{0} u_{1} u_{1}^{\prime}\right)+M_{23}\left(u_{0} u_{1}^{\prime 2}+2 u_{0}^{\prime} u_{1} u_{1}^{\prime}\right)+M_{24}\left(u_{0}^{\prime \prime} u_{1}^{2}+2 u_{0} u_{1} u_{1}^{\prime \prime}\right)\right. \\
& \left.\left.+2 M_{25} u_{0} u_{1}\right] d s+\int_{0}^{s_{1}}\left(M_{21} u_{1}^{3}+M_{22} u_{1}^{2} u_{1}^{\prime}+M_{23} u_{1} u_{1}^{\prime 2}+M_{24} u_{1}^{2} u_{1}^{\prime \prime}+M_{25} u_{1}^{2}\right) d s\right\},
\end{aligned}
$$

where

$$
\left.\left.M_{21}=-r\left(\beta^{\prime 3}+\frac{z^{\prime 3}}{r^{3}}\right), \neg M_{22}=-2 \frac{r^{\prime} z^{\prime}}{r},\right\urcorner M_{23}=-\frac{1}{2} r\left(\beta^{\prime}-\frac{z^{\prime}}{r}\right),\right\urcorner M_{24}=-2 r \beta^{\prime}, \quad M_{25}=-\frac{1}{2} q_{0} r .
$$

The coefficients $A_{3}$ and $B_{3}$ of the equation (41) are

$$
\begin{aligned}
A_{3}= & \frac{1}{G_{1}} r^{2}\left(s_{1}\right)\left|z\left(s_{1}\right)\right| E_{3}, \\
B_{3}= & \int_{0}^{s_{1}}\left(2 M_{31} b_{3}+2 M_{32} b_{3}^{\prime}+2 M_{33} b_{3}^{\prime \prime}+M_{34}\right) d s+2 \alpha_{3} \int_{0}^{s_{1}}\left(M_{31} u_{0}+M_{32} u_{0}^{\prime}+M_{33} u_{0}^{\prime \prime}\right) d s \\
& -\frac{2 E_{3}}{G_{1}}\left[\int_{0}^{s_{1}} b_{3} r d s+\alpha_{3} \int_{0}^{s_{1}} u_{0} r d s-\frac{1}{2} q_{0} \int_{0}^{s_{1}} r r^{\prime 2} d s\right],
\end{aligned}
$$

where

$$
\begin{aligned}
& E_{3}=\int_{0}^{s_{1}}\left(M_{31} u_{1}+M_{32} u_{1}^{\prime}+M_{33} u_{1}^{\prime \prime}\right) d s+\alpha_{1} \int_{0}^{s_{1}}\left(M_{31} u_{0}+M_{32} u_{0}^{\prime}+M_{33} u_{0}^{\prime \prime}\right) d s, \\
& M_{31}=-r^{\prime 2} z^{\prime}\left(\beta^{\prime}-\frac{z^{\prime}}{r}\right)^{2}, \neg M_{32}=-\frac{r^{\prime} z^{\prime}}{r}\left[r^{\prime 2}-\frac{1}{2} r^{2} \beta^{\prime}\left(\beta^{\prime}-\frac{z^{\prime}}{r}\right)\right], \neg M_{33}=-r r^{\prime 2} \beta^{\prime}, \\
& M_{34}=-r r^{\prime 4}\left(\frac{z^{\prime 4}}{r^{4}}-2 \beta^{\prime 4}\right)+r^{\prime 4} z^{\prime} \beta^{\prime}\left(\frac{z^{\prime 2}}{r^{2}}+7 \frac{z^{\prime}}{r} \beta^{\prime}-5 \beta^{\prime 2}\right)+r r^{\prime 2} z^{\prime 2} \beta^{\prime 2}\left(-\frac{z^{\prime 2}}{r^{2}}+2 \frac{z^{\prime}}{r} \beta^{\prime}-3 \beta^{\prime 2}\right),
\end{aligned}
$$

the function $b_{3}(s)$ is the solution of the problem

$$
L_{0} b_{3}=F_{3}(s) \quad\left(0 \leqslant s \leqslant s_{1}\right), \neg b_{3}(0)=1, \neg b_{3}^{\prime}(0)=0,
$$

and $\alpha_{3}=-b_{3}\left(s_{1}\right) / u_{0}\left(s_{1}\right)$. 
${ }^{1}$ J. Plateau, Statique Expérimentale et Théoretique des Liquides (GautierVillars, Paris, 1873)

${ }^{2}$ I. Martínez, J. M. Haynes, and D. Langbein, "Fluid statics and capillarity," in Fluid Sciences and Material Sciences in Space (Springer-Verlag, Berlin, 1987), pp. 53-80.

${ }^{3}$ B. J. Lowry and P. H. Steen, "Capillary surfaces: stability from families of equilibria with application to the liquid bridge," Proc. R. Soc. London A 449, 411 (1995).

${ }^{4}$ D. Langbein, "Crystal growth from liquid columns," J. Cryst. Growth 104, 47 (1990).

${ }^{5} \mathrm{G}$. Mason, "An experimental determination of the stable length of cylindrical liquid bubbles," J. Colloid. Interface Sci. 32, 172 (1970).

${ }^{6}$ Lord Rayleigh, "On the capillary phenomena of jets," Proc. R. Soc. London 29, 71 (1879).

${ }^{7}$ R. D. Gillette and D. C. Dyson, "Stability of fluid interfaces of revolution between equal solid circular plates," Chem. Eng. J. 2, 44 (1971).

${ }^{8}$ D. Rivas and J. Meseguer, "One-dimensional self-similar solution of the dynamics of axisymmetric slender liquid bridges," J. Fluid Mech. 138, 417 (1984)

${ }^{9}$ L. A. Slobozhanin, "Problems of stability of an equilibrium liquid encountered in space technology research,"' in Fluid Mechanics and Heat/ and Mass Transfer Under Zero-Gravity (Nauka, Moscow, 1982), pp. 9-24 (in Russian).

${ }^{10}$ A. D. Myshkis, V. G. Babskii, N. D. Kopachevskii, L. A. Slobozhanin, and A. D. Tyuptsov, Low-Gravity Fluid Mechanics (Springer-Verlag, Berlin, 1987).

${ }^{11}$ M. A. Erle, R. D. Gillette, and D. C. Dyson, "Stability of interfaces of revolution with constant surface tension. The case of catenoid," Chem. Eng. J. 1, 97-109 (1970).

${ }^{12}$ W. Howe, Ph.D. Dissertation, Friedrich-Wilhelms Universität zu Berlin, 1887.

${ }^{13}$ R. D. Gillette and D. C. Dyson, "Stability of axisymmetric liquid-fluid interfaces towards general disturbances," Chem. Eng. J. 3, 196 (1972).

${ }^{14}$ L. A. Slobozhanin and J. M. Perales, "Stability of liquid bridges between equal disks in an axial gravity field," Phys. Fluids A 5, 1305 (1993).

${ }^{15}$ M. P. Elagin, A. P. Lebedev, and A. V. Shmelev, "Laboratory modelling of the stability and dynamics of free liquid zones," in Fluid Mechanics and Heat/and Mass Transfer Under Zero-Gravity (Nauka, Moscow, 1982), pp. 24-33 (in Russian).

${ }^{16} \mathrm{~A}$. Sanz and I. Martínez, "Minimum volume for a liquid bridge between equal disks," J. Colloid. Interface Sci. 93, 235 (1983).
${ }^{17}$ M. J. Russo and P. H. Steen, "Instability of rotund capillary bridges to general disturbances: experiment and theory," J. Colloid. Interface Sci. 113, 154 (1986)

${ }^{18}$ L. A. Slobozhanin and A. D. Tyuptsov, "Stable equilibrium of the surface of a capillary liquid in contact with the edge of a solid,' Izv. Akad. Nauk SSSR. Mekh. Zhid. Gaza 1, 3 (1974) (in Russian); English translation: Fluid Dynam. 9(1), 1 (1974).

${ }^{19}$ L. A. Slobozhanin, "Some problems of stability of zero- $g$ liquid bridges," in Hydromechanics and Heat/Mass Transfer in Microgravity (Gordon and Breach, New York, 1992), pp. 185-192.

${ }^{20}$ R. A. Brown and L. E. Scriven, "The shapes and stability of captive rotating drops," Philos. Trans. R. Soc. London A 297, 51 (1980).

${ }^{21}$ L. H. Ungar and R. A. Brown, "The dependence of the shape and stability of captive rotating drops on multiple parameters,' Trans. R. Soc. London A 306, 347 (1982).

${ }^{22}$ J. Tsamopolous, A. J. Polinski, and M. E. Ryan, "Equilibrium shapes and stability of captive annular menisci," J. Fluid Mech. 197, 523 (1988).

${ }^{23}$ A. D. Tyuptsov, A. D. Myshkis, and L. A. Slobozhanin, "'General solution procedure for problems of small perturbations and bifurcations of equilibrium capillary liquid surfaces," Micrograv. Sci. Technol. VII/1, 24 (1994).

${ }^{24}$ D. H. Michael, "Meniscus stability," Ann. Rev. Fluid Mech. 13, 189 (1981).

${ }^{25}$ J. M. Vega and J. M. Perales, "Almost cylindrical isorotating liquid bridges for small Bond numbers," in Materials Sciences Under Microgravity (ESA SP-191, 1983), pp. 247-252.

${ }^{26}$ J. Meseguer, "The breaking of axisymmetric liquid bridges," J. Fluid Mech. 130, 123 (1983).

${ }^{27}$ J. Meseguer, A. Sanz, and D. Rivas, "The breaking of axisymmetric noncylindrical liquid bridges," in Materials Sciences Under Microgravity (ESA SP-191, 1983), pp. 261-265.

${ }^{28}$ D. J. Struik, Classical Differential Geometry (Addison-Wesley, Reading, MA, 1957).

${ }^{29}$ A. A. Fowle, J. S. Haggarty, R. R. Perron, P. F. Strong, and J. R. Swanson, "Float-zone processing in a weightless environment," NASA CR-2768, Washington, DC, 1976.

${ }^{30}$ M. M. Vainberg and V. A. Trenogin, Theory of Branching of Solutions of Non-Linear Equations (Noordhoff, Leyden, 1974). 
Physics of Fluids is copyrighted by AIP Publishing LLC (AIP). Reuse of AIP content is subject to the terms at: http://scitation.aip.org/termsconditions. For more information, see http://publishing.aip.org/authors/rights-and-permissions. 